\title{
Vertical Shear Instability in the Solar Nebula
}

\author{
Min-Kai Lin and Andrew N. Youdin \\ Department of Astronomy and Steward Observatory, \\ University of Arizona, 933 North Cherry Avenue, Tucson, AZ 85721, USA \\ email: minkailin@email.arizona.edu, youdin@email.arizona.edu
}

\begin{abstract}
We quantify the thermodynamic requirement for the Vertical Shear Instability and evaluate its relevance to realistic protoplanetary disks as a potential route to hydrodynamic turbulence.
\end{abstract}

Keywords. accretion, accretion disks, hydrodynamics, instabilities, methods: analytical

\section{Introduction}

A purely hydrodynamic route to turbulence has important implications for the evolution of cold protoplanetary disks and solids within them. One such candidate is the Vertical Shear Instability (VSI) operating in disks where the orbital frequency $\Omega$ depends on the height $z$ away from the disk midplane. While astrophysical disks generally possess vertical shear, VSI also requires short thermal timescales. We determine a quantitative thermodynamic requirement for the VSI, and apply our results to realistic disk conditions. We find the VSI can operate effectively at 5-50AU in a typical protoplanetary disk, with characteristic growth times of 30 orbits.

\section{The need for rapid cooling for the VSI}

A radial variation in a disk's entropy or temperature profile leads to vertical shear, $\partial_{z} \Omega \neq 0$. This is a source of free energy, and may thus lead to instability (Goldreich \& Schubert 1967; Urpin 2003; Barker \& Latter 2015), provided the vertical lengthscale of the disturbance is much larger than its radial lengthscale. However, in stably stratified, irradiated protoplanetary disks (Chiang \& Youdin 2010), the VSI also requires rapid cooling to overcome the stabilizing influence of vertical buoyancy (Nelson et al. 2013).

More specifically, for a vertically isothermal disk with radial temperature dependence $T \propto r^{q}$, the cooling or thermal relaxation timescale $t_{c}$ must be sufficiently small for the VSI to operate effectively:

$$
t_{c} \Omega_{\mathrm{K}}<\frac{h|q|}{\gamma-1}
$$

where $\Omega_{\mathrm{K}}$ is the Keplerian frequency, $h$ is the disk aspect ratio, and $\gamma$ is the adiabatic index (Lin \& Youdin 2015). Since protoplanetary disks are typically thin, $h \ll 1$, Eq. 1 implies that $t_{c} \ll \Omega_{\mathrm{K}}$ is required for the VSI. That is, the thermal timescale must be significantly shorter than the dynamical timescale, which reflects the fact that vertical shear is weakly destabilizing, while vertical buoyancy is strongly stabilizing.

\section{Applicability of the VSI in realistic protoplanetary disks}

We solve the hydrodynamic linear stability problem for axisymmetric perturbations in a disk model of the Solar Nebula described in Chiang \& Youdin (2010). We include in 


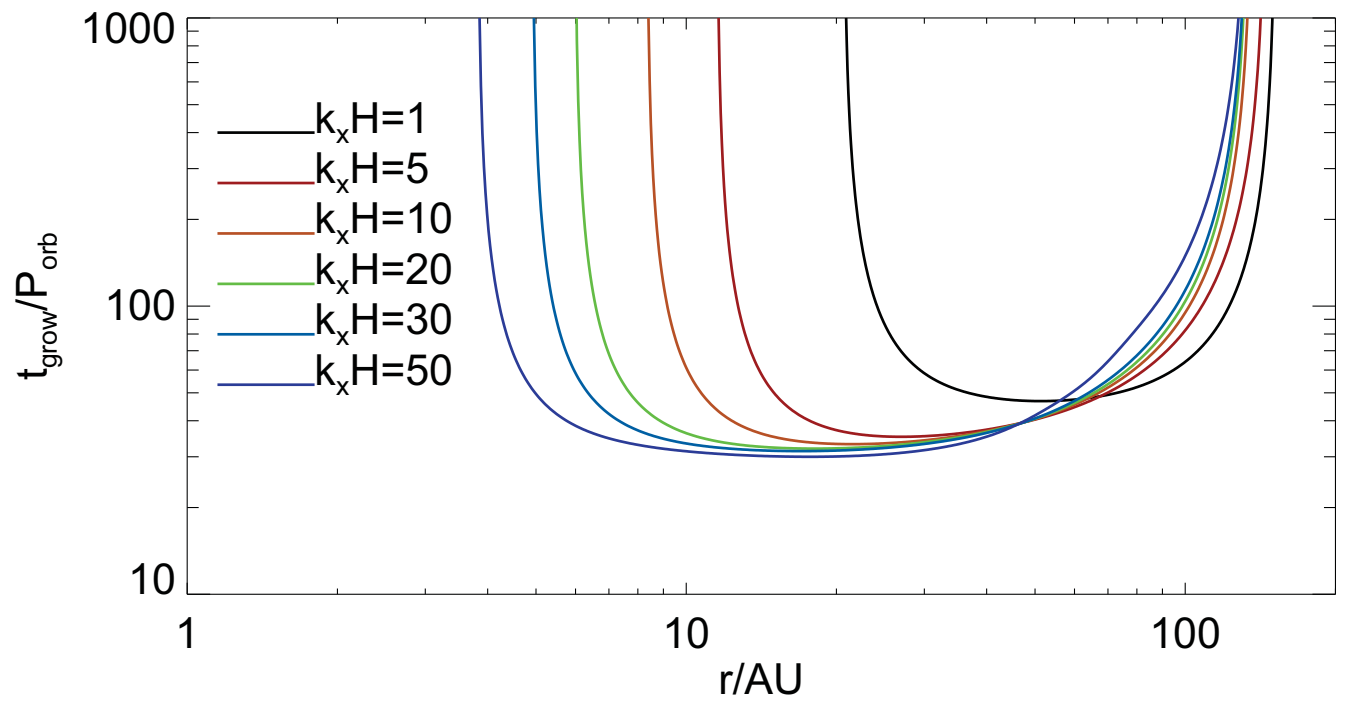

Figure 1. Characteristic growth times of the Vertical Shear Instability in a typical protoplanetary disk model. Here, $k_{x}$ is the radial wavenumber of the disturbance under consideration and $H$ is the disk scale-height.

the energy equation a realistic cooling function based on dust opacity, which depends on $z$ as well as the disturbance lengthscale. (For details, see Lin \& Youdin 2015.)

In Fig. 1 we plot characteristic VSI growth times for a range of radial perturbation wavenumbers. Growth times increase rapidly at $\lesssim 5 \mathrm{AU}$ and $\gtrsim 100 \mathrm{AU}$ as the cooling times violate Eq. 1 in these regions. Thus, the VSI is most effective at intermediate radii. Notice also the growth times are strongly dependent on lengthscale toward the opticallythick inner disk, with smaller-scale disturbances being able to operate further inwards. In the optically-thin outer disk, cooling times are independent of $k_{x}$ and all scales are stabilized beyond approximate the same radius.

\section{Conclusions}

Vertical shear is ubiquitous in astrophysical disks. Such configurations are unstable if the cooling timescale is significantly shorter than the dynamical timescale (Eq. 1) in a thin disk. This can be satisfied for small-scale disturbances at 10 s of AU in typical protoplanetary disk models with realistic cooling. We have thus shown that the VSI is dynamically important in the outer parts of protoplanetary disks. This is expected to have significant implications for mass transport and dust evolution in these regions.

\section{References}

Barker, A. J. \& Latter, H. N. 2015, MNRAS, 450, 21

Chiang, E. \& Youdin, A. N. 2010, Annual Review of Earth and Planetary Sciences, 38, 493

Goldreich, P. \& Schubert, G. 1967, ApJ, 150, 571

Lin, M.-K. \& Youdin, A. N. 2015, ApJ, submitted, ArXiv: 1505.02163

Nelson, R. P., Gressel, O., \& Umurhan, O. M. 2013, MNRAS, 435, 2610

Urpin, V. 2003, A\& A, 404, 397 